\title{
POLARIZATION CORRELATIONS IN THE SLC FINAL FOCUS
}

\author{
F.-J. Decker, Stanford Linear Accelerator Center*, Stanford University, Stanford, CA 94309 USA
}

\begin{abstract}
At the SLC, the electron beam polarization $(\mathrm{P})$ is measured by a Compton polarimeter downstream of the Interaction Point. This measurement averages over the entire beam distribution and must be corrected for various correlations to calculate the luminosity (L) weighted polarization. Because the spin rotation in the ARC is energy dependent, off energy particles have lower polarization. These particles may also be poorly focused and contribute less luminosity due to the higher order chromatic optics of the final focus. The small vertical $\beta$ function at the interaction point also causes an hour-glass effect, where particles at the head and tail of the bunch have less luminosity. Since there is an energy-z correlation due to the compensation of longitudinal wakefields in the linac, these particles may also have lower polarization. The correlations are: $\mathrm{z} \longrightarrow \mathrm{E}$ (linac), $\mathrm{E} \longrightarrow \mathrm{P}($ ARC), $\mathrm{z} \longrightarrow$ $\mathrm{L}$ (hour-glass) and $\mathrm{E} \longrightarrow \mathrm{L}$ (chromaticity). The contributions from the $\mathrm{z} \longrightarrow \mathrm{E}$ and $\mathrm{z} \longrightarrow \mathrm{L}$ correlations are discussed.
\end{abstract}

\section{INTRODUCTION}

At the Stanford Linear Collider (SLC) highly polarized (80\%) electrons collide with positrons at the $Z$ mass. The asymmetry in the measured cross section for left and right handed electrons $\left(A_{L R}\right)$ determines important high energy parameters like $\sin ^{2} \theta_{w}$ and the top mass range [1]. The statistical error of the $A_{L R}$ up to this year is about $\pm 3 \%$ and the systematic around $1.5 \%$. Any additional systematics should be carefully checked. The big correction of about 2\% for a low energy tail in 1993 [2] is much reduced for the 94/95 run since there are less low energy tails due to over-compression [3]. The number of effective spin rotations in the ARCs was not reduced conciderably (from 17 to 13 turns), so other effects have to be investigated. Here we will discuss the effect of the energy spread, which has a certain correlation with the $z$ distribution due to longitudinal wakefields in the linac. This longitudinal distribution correlates with the luminosity at the interaction point (IP), due to the hour-glass effect, where earlier and later particles contribute less to the luminosity. Since these particles are off in energy and the effective polarization of the collisions will be slightly higher.

*Work supported by the Department of Energy, contract DE-AC03-76SF00515.

\section{ENERGY-Z CORRELATION}

The correlation for the energy in the longitudinal dimensions comes mainly from the rf sinus curve and the longitudinal wakefield. By chosing the bunch length to about $1.2 \mathrm{~mm}(\sigma)$ at $3.5 \cdot 10^{10}$ particles per bunch, the generated wakefield compensates the rf sinus curve roughly. The bunch shaping with over-compression reduced the long low energy tails, so that the energy distribution was about Gaussian with an energy spread of $0.10-0.15 \%$. Since the intrinsic energy spread is about $0.04 \%$, there is some correlation with $z$. The front has normally a higher energy than the back. This leads also to a compression in the ARCS. A term of $R_{56}=150 \mathrm{~mm}$ gives about a compression of $0.15-0.23 \mathrm{~mm}$ or an IP bunch length of $1.0 \pm 0.05 \mathrm{~mm}$ at the IP.

A simulation of the longitudinal wakefield effect showed a big sensitivity to the overall phase of the linac. Small variations of $1^{\circ}$ in phase will vary the bunch length at the IP by $20 \%$ (see Fig. 1).

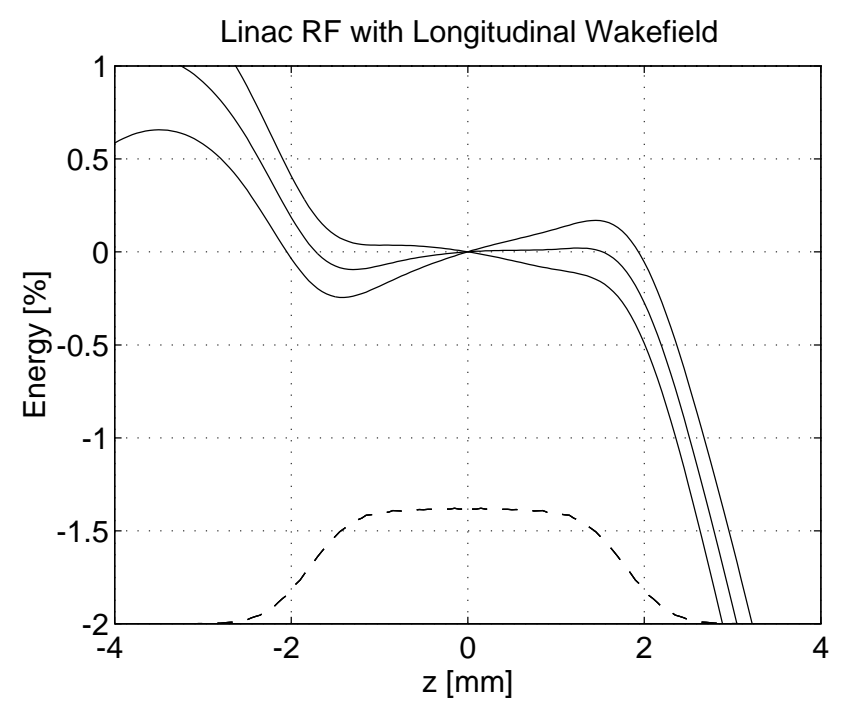

Fig. 1: Energy-z correlation for different linac phase. Small phase changes of $\pm 1^{\circ}$ influence the energy- $z$ correlation which changes the IP bunch length. The beam distribution (dashed) is generated by over-compression.

\section{HOUR-GLASS EFFECT}

The strong focussing of the beams at the IP result in an beam envelope which has the form of an hour-glass. 


\section{A. Description}

At the IP there are two effects which produce different luminosity for different longitudinal positions. One is the hour-glass effect, where particles in the head and the tail of the bunch collide mainly past or in front of the IP with the smallest spot size. The other is the disruption, where the strong beam-beam forces bend the beams towards each other shifting the beam waist somewhat downstream. More sophisticated correlations like traveling focusing, where different longitudinal parts of the bunch have their focus at different $z$ locations, are not considered in this paper.

The hour-glass effect comes mainly from $y$. With an IP beam size of $0.5 \mu \mathrm{m}$ and an angular divergence of 250 $\mu \mathrm{rad}$, the spot size at $z=0.5 \mathrm{~mm}$ (one effective sigma away) will be $0.515 \mu \mathrm{m}$ or $3 \%$ bigger $\left(12 \%\right.$ at $\left.2 \sigma_{z e f f}\right)$ :

$$
\sigma_{y}=\sqrt{\sigma_{y o}^{2}+\left(\theta_{y} z\right)^{2}} .
$$

Figure 2 shows the behavior of the this formula with a correction for the disruption effect.

Hour Glass Effect, 1.0 mm, 250 urad, 0.5 um

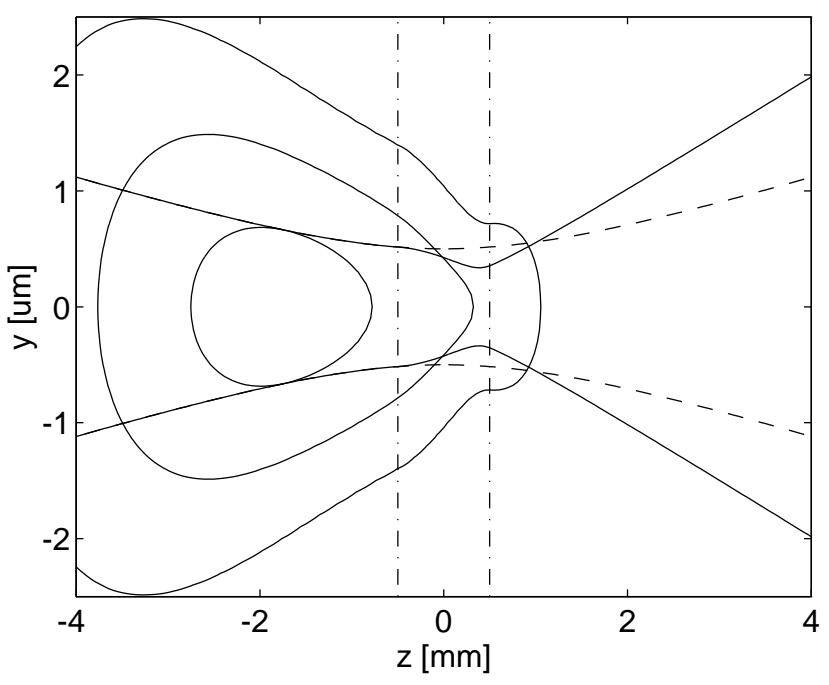

Fig. 2: Hour glass effect with disruption.

The beam enters from the left with a $y$ - $z$ distribution indicated by $a 1,2$, and 3 sigma contour lines. The beambeam forces from the other bunch were assumed to be constant over $\pm \sigma_{z} / 2$ (dash-dotted line). The smaller spot and the bigger outgoing angular divergence is visible from the 1-sigma envelope lines. The dashed curve is the not disrupted case.

The beam-beam disruption [4] or pinch effect focuses the beam even further. The approximation was used, that the forces were locally fixed and change the beam match in the following way:

$$
\begin{aligned}
& \sigma_{y o d}=\sqrt{\sigma_{y o}^{2} \cdot \cos ^{2}(k z)+\theta_{y o}^{2} \cdot \sin ^{2}(k z) / k^{2}} \text { and } \\
& \theta_{y d}=\theta_{y} \cdot \sigma_{y} / \sigma_{y o d} \text { for }-\sigma_{z}<2 z<\sigma_{z} \\
& \text { with } k=\sqrt{\frac{k_{b}}{\sigma_{z}}} \text { and } k_{b}=\frac{633 \cdot N / 10^{10}}{\sigma_{y}\left(\sigma_{x}+\sigma_{y}\right)} .
\end{aligned}
$$

The spot size in $x$ is $2.1 \mu \mathrm{m}$ and $N=3.5 \cdot 10^{10}$. The beam spot is reduced while the angular divergence increases, keeping the emittance constant in this linarized approach.

\section{B. Estimate}

The effective turn in the ARCs set the scale of the problem. With 25 turns and 1\% energy offset the spin of these particles will rotate 0.25 turns or $90^{\circ}$, which means they would not have any longitudinal polarization at the IP. For $n=13$ turns and a $\Delta E / E=0.3 \%$ energy offset the loss in polarization is $3 \%$ :

$$
\Delta P=1-\cos \left(2 \pi n \frac{\Delta E}{E}\right)
$$

Since there are not too many particles out there the correction will be small. If all the particles outside of 2 sigma $(4.6 \%)$ would be about $4 \%$ off in polarization, but still giving about $80 \%$ of the IP luminosity, the effect would cause only a correction of $0.04 \%$. The highly nonlinear behavior (quadratic in $E$ and $z$ ) can give bigger values especially if the cosine curves are not perfectly centered. To quantify the effect a small simulation program was written.

\section{Simulations}

The energy spread is assumed to be Gaussian and totally correlated with $z$. The energy spread is $0.15 \%$ and the effective bunch length at the IP is $0.5 \mathrm{~mm}$, half the real value and totally correlated with energy (assumption). The angular divergence in $y$ is $250 \mu \mathrm{rad}$ and the spot size 0.5 $\mu \mathrm{m}$. Due to the hour-glass effect (Eq. 1) the spot is bigger and will give less luminosity (3\%). An off-energy particle will be sitting off the core and gives even less luminosity. Fig. 3 shows the luminosity versus energy offset and gives for comparison the chromatic effect of $U_{3246}=850 \mathrm{~m}$. 


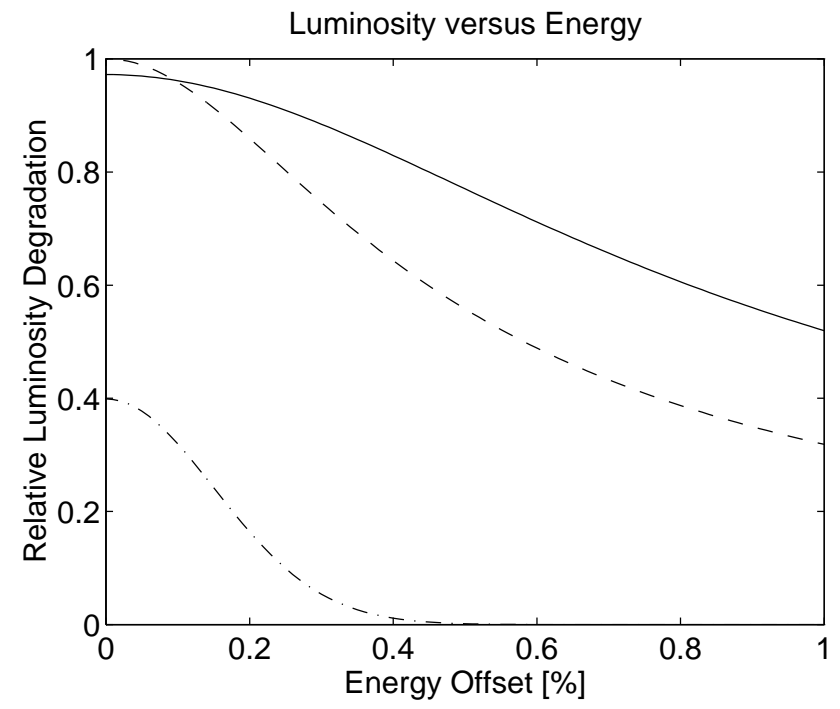

Fig. 3: Luminosity versus Energy Offset

The hour-glass effect (solid) on the luminosity versus energy is less than the direct chromatic effect from a higher order term (dash). A typical ( $0.15 \%)$ energy distribution is given dash-dotted.

\section{HIGHER ORDER CHROMATICS}

For the 1994/95 run the higher order chromatics consist mainly of the term $U_{3246}=850 \mathrm{~m}$. The effect on the spot size is as follow:

$$
\sigma_{y}=\sqrt{\sigma_{y o}^{2}+\left(2 \cdot U_{3246} \cdot \theta_{x} \cdot \theta_{y} \cdot \delta\right)^{2}}
$$

where the initial size is enlarged by a term which depends on the angular divergences in $x(350 \mu \mathrm{rad})$ and $y$, and the energy spread $\delta$.

\section{DISCUSSION OF RESULTS}

The hour-glass and direct chromatic effect on the polarization at the interaction point give small corrections of $0.04 \%$ and $0.09 \%$. For 17 turns the chromatic number is $0.16 \%$ which is half the value calculated a year ago with a different program and somewhat different parameters, e.g. real, non-gaussian bunch distribution in $z$. with more weight in the tails. Taking into account the quadratic effects and any mistuning an overall corrections of about plus $0.2 \pm 0.1 \%$ from the apparently lost polarization of $-0.75 \%$ from the ARCs seems reasonable.

\section{ACKNOWLEDGMENT}

I would like to thank M. Fero for his interest and his careful reading of the manuscript.

\section{REFERENCES}

[1] SLD Collaboration, Precise Measurement of the Left-Right Cross Section Asymmetry in Z Boson Production by $e^{+} e^{-}$Collisions, Phys. Rev. Letters, 4 July 1994, Vol. 73, Number 1, p. 25.

[2] F.-J. Decker, J.T. Seeman, Luminosity Polarization Correlation in the SLC, EPAC, London, June 94.

[3] F.-J. Decker, R. Holtzapple, T. Raubenheimer, Over-Compression, a Method to Shape The Longitudinal Bunch Distribution for a Reduced Energy Spread, LINAC94, Tsukuba, Aug. 1994, p. 47.

[4] P. Raimondi, P. Chen, F.-J. Decker, Disruption Effects on the Beam Size Measurement, PAC95, Dallas, May 1995. 